\title{
Study Requirement Object
}

National Cancer Institute

\section{Source}

National Cancer Institute. Study Requirement Object. NCI Thesaurus. Code C45320.

A dimension table that lists the biospecimen requirements for this ST UDY Biospecimen

requirements include: . (caTISSUE) 